\title{
Objective and Subjective Socioeconomic Status and Susceptibility to the Common Cold
}

\author{
Sheldon Cohen \\ Carnegie Mellon University \\ Nancy Adler \\ University of California
}

\author{
Cuneyt M. Alper and William J. Doyle \\ Pittsburgh Children's Hospital and University of Pittsburgh \\ School of Medicine \\ John J. Treanor \\ University of Rochester School of Medicine and Dentistry
}

\author{
Ronald B. Turner \\ University of Virginia Health Sciences Center
}

\begin{abstract}
Objective: We ask whether subjective socioeconomic status (SES) predicts who develops a common cold when exposed to a cold virus. Design: 193 healthy men and women ages 21-55 years were assessed for subjective (perceived rank) and objective SES, cognitive, affective and social dispositions, and health practices. Subsequently, they were exposed by nasal drops to a rhinovirus or influenza virus and monitored in quarantine for objective signs of illness and self-reported symptoms. Main Outcome Measures: Infection, signs and symptoms of the common cold, and clinical illness (infection and significant objective signs of illness). Results: Increased subjective SES was associated with decreased risk for developing a cold for both viruses. This association was independent of objective SES and of cognitive, affective and social disposition that might provide alternative spurious (third factor) explanations for the association. Poorer sleep among those with lesser subjective SES may partly mediate the association between subjective SES and colds. Conclusions: Increased Subjective SES is associated with less susceptibility to upper respiratory infection, and this association is independent of objective SES, suggesting the importance of perceived relative rank to health.
\end{abstract}

Keywords: subjective socioeconomic status, infection, psychoneuroimmunology, common cold, susceptibility

Increasing socioeconomic status (SES), whether measured in terms of income, education or occupation, has been associated with decreasing rates of mortality and morbidity from almost every disease condition (e.g., Adler et al., 1994; Anderson \& Armstead, 1995; Marmot, Feeney, Shipley, North, \& Syme, 1995). This

Sheldon Cohen, Department of Psychology, Carnegie Mellon University; Cuneyt M. Alper and William J. Doyle, Department of Otolaryngology, Pittsburgh Children's Hospital and University of Pittsburgh School of Medicine; Nancy Adler, Department of Psychiatry, University of California, San Francisco; John J. Treanor, Infectious Disease Unit, University of Rochester School of Medicine and Dentistry; Ronald B. Turner, Department of Pediatrics, University of Virginia Health Sciences Center.

Research reported here was funded by grants to the Pittsburgh MindBody Center from the National Heart, Lung and Blood Institute (HL65111; HL65112) and by supplementary funds provided by the John D. and Catharine T. MacArthur Foundation Network on Socioeconomic Status and Health. The influenza virus was provided by the National Institute of Allergy and Infectious Diseases, Division of Microbiology and Infectious Diseases. The authors are indebted to Jeffrey Best, Wesley Barnhart and Ellen Conser for their assistance in preparing this paper.

Correspondence concerning this article should be addressed to Sheldon Cohen, Department of Psychology, Carnegie Mellon University, Pittsburgh, PA 15213. E-mail: scohen@cmu.edu relationship exists across countries, with and without universal access to health care, suggesting that access to care is not the primary mechanism behind this effect.

One issue raised by this literature is whether these associations are attributable to differences in material resources associated with these SES markers or to the perceptions of relative social status that they are thought to generate. Because the literature associating SES with morbidity and mortality is based on objective markers of SES, it does not allow us to differentiate the potential effects of resources versus those of perceived rank. However, recently, a new instrument has been applied in an attempt to tap people's perceptions of how they rank in SES in relation to others. The instrument is a simple picture of a ladder with 9 or 10 steps. The respondent is asked to place themselves on the ladder in terms of where they stand in their country, in terms of income, education and occupation (Adler et al., 1994). It is possible that one's subjective relative rank is more important than the environmental exposures and resources represented by objective measures of SES (e.g., animal studies of social rank where environments and resources are controlled: Cohen et al., 1997b; Kaplan, Manuck, Clarkson, Lusso \& Taub, 1982). If so, the ladder would predict health above and beyond the objective markers.

A number of studies across different populations have found that higher subjective SES, as assessed by the ladder, is associated 
with better health and that these relations generally remain after controlling for traditional objective measures of SES (Adler, Epel, Castellazzo, \& Ickovics, 2000; Hu, Adler, Goldman, Weinstein, \& Seeman, 2005; Ostrove, Adler, Kuppermann, \& Washington, 2000; Singh-Manoux, Adler, \& Marmot, 2003). These studies are all cross-sectional. Moreover, with two exceptions, they used measures of health outcomes that were self-reported. The exceptions are Adler et al. (2000), who found preliminary associations between lower subjective social status and several neuroendocrine mediators of disease risk, and Wright and Steptoe (2005), who reported a relation between low social status and an exaggerated rise in cortisol on awakening. Here we present evidence on the associations of objective and subjective SES and health using a prospective design and objective markers of disease as the outcome. We assessed income, education, and subjective SES in healthy volunteers and subsequently exposed them to one of two viruses that cause a common cold. We ask whether the objective and subjective measures of SES predict who develops a cold, and whether subjective SES predicts disease susceptibility above and beyond objective markers. In additional analyses, we control for a range of personality characteristics that might contribute both to judgments of subjective SES and to disease resistance and control for health practices that might mediate an association between subjective SES and resistance.

\section{Method}

\section{Design}

After we assessed objective and subjective SES, demographics, personality characteristics, health practices, and virus-specific antibody levels, healthy volunteers were quarantined in separate rooms, exposed to either a rhinovirus (RV) or influenza virus, and followed for five (for RV) or six (for influenza virus) days to assess infection, and signs and symptoms of illness.

\section{Subjects}

Data were collected between 2000 and 2004. The subjects were 95 men and 98 women, aged 21 to 55 years $(M=37.3, S D=$ \pm 8.8 ) who responded to advertisements and were judged to be in good health. They were studied in eleven groups and were paid $\$ 800$ for their participation. The study received institutional review board approval and informed consent was obtained from each subject.

\section{Experimental Plan}

Volunteers underwent medical screenings and were excluded if they had a history of nasal surgery, asthma, or cardiovascular disorders, or abnormal urinalysis, CBC, or blood enzymes, were pregnant or currently lactating, seropositive for HIV, or on regular medication. They were also excluded if they were hospitalized for psychiatric problems during the last five years or were currently taking medications for psychiatric problems. Those in influenza virus trials also had baseline electrocardiograms and were excluded if there were any abnormal findings.

Specific serum antibody titer to the challenge virus, demographics, weight and height were assessed at screening. To maximize the rate of infection, only subjects with viral-specific antibody titers $\leq$
4 were included in the study. SES, personality and health practice measures were assessed during the six weeks between screening and virus exposure.

During the first 24 hours of quarantine (before viral exposure), volunteers had a nasal examination and a nasal lavage. Baseline symptoms, nasal mucociliary clearance, and nasal mucus production were assessed. Volunteers were excluded at this point if they had signs or symptoms of a cold and data for subjects were excluded from the analyses if a viral pathogen was isolated from the nasal lavage obtained at that time.

Subjects were then given nasal drops containing 125 Tissue Culture Infectious Dose ${ }_{50}\left(\mathrm{TCID}_{50}\right)$ of RV39 [N $\left.=155\right]$ or $10^{5}$ $\mathrm{TCID}_{50}$ of influenza A/Texas/36/91 [N = 38]. Disease expression in both viruses is a common cold-like upper respiratory illness. We used two virus types in order to establish the generalizability of any observed associations. On each day of quarantine, volunteers recorded their respiratory symptoms, were assessed for nasal mucociliary clearance and nasal mucus production, and nasal lavage samples were collected for virus culture. Approximately 28 days after virus exposure, blood was collected for serological testing. The investigators were blinded to all psychological, health practice, and biological measures.

\section{Socioeconomic Status}

We employed two objective measures of SES: income and education.

Income. Income was assessed by the question, "Which category best describes your yearly household income before taxes?" There were 13 categories ranging from "less than $\$ 5,000 "$ to "\$150,000 or more." The categories were narrower in the bottom range of incomes ( $\$ 5,000$ increments) and progressively increased with increases in income (e.g., $\$ 10,000$ at $\$ 30-\$ 50$ thousand and $\$ 25,000$ increments at $\$ 100,000$ and above). "Income" was defined as the median income of the identified category. The income score was log transformed to better approximate a normal distribution.

Education. Education was assessed by the question, "What is the highest grade or year of school you have completed?" There were 18 categories ranging from "no formal education" to "doctoral degree (PhD, MD, EdD, DVM, DDS, JD, etc.). Subjects were assigned a number of years of education based on their response (e.g., high school education $=12$ years, associates degree $=14$ years, and a $\mathrm{PhD}=20$ ).

Subjective SES. Subjects were presented with a picture of a 9-rung ladder. They were ask to place themselves on the ladder (check a rung) based on where they stand compared to other persons in the United States in terms of income, education, and occupation (Adler et al., 1994). They were assigned scores ranging from 1 (lowest rung) to 9 (highest rung).

\section{Standard Control Variables}

In the analyses, we control for the effects of immunity to the virus as assessed by pre-challenge antibody titer (within virus), age, body mass index (BMI: weight [kilograms]/height $[\text { meters }]^{2}$ ), race (Caucasian, African-American, other), sex, virus-type (influenza or RV), and season of exposure (spring, summer, autumn, winter). 


\section{Controlling for Emotional Styles}

We have published results from this data set indicating that higher levels of positive emotional style (trait positive affect) were associated with less susceptibility to disease (Cohen, Alper, Doyle, Treanor, \& Turner, 2006). To establish the independence of effects reported here, we included both positive and negative emotional style measures as controls. Volunteers were interviewed by phone on 14 consecutive evenings during the month before quarantine. They were asked how accurately $(0=$ not at all accurate to $4=$ extremely accurate) each of 6 positive and 6 negative adjectives described how they felt during the last day (see details in Cohen et al., 2006). Daily positive and negative mood scores were calculated by summing the ratings of the 6 respective adjectives. To form summary measures of emotional style, daily mood scores were averaged (separately for positive and negative) across the 14 days.

\section{Potential Confounding Variables}

We assessed several personality characteristics that might bias one's perceptions of his/her own social status and influence health outcomes. These included mastery, optimism, self-esteem, purpose, and extraversion. The 7-item Mastery Scale (Pearlin \& Schooler, 1978) was used to assess the extent to which one feels as though they manifest personal mastery over important life outcomes. The 10-item Life Orientation Test-R (Scheier, Carver, \& Bridges, 1994) was used to assess dispositional optimism; the 4-item version of the Rosenberg Self-esteem Scale (Rosenberg, 1965) was used to assess self-esteem; and the 6-item Life Engagement Test (Scheier et al., 2006) was used to assess the extent to which a person is purposefully engaged in the current activities of life. On all four scales, respondents indicated how much they agreed or disagreed with self-descriptive sentences. No time frame or reference periods were used. For all the scales, the appropriate items were reversed and the scale scores were summed. The internal reliabilities were .72 for mastery, .78 for optimism, .84 for self-esteem, and .73 for purpose.

Extraversion was assessed with the 5-item version of the extraversion subscale from the Goldberg Big Five Questionnaire (Goldberg, 1992; Cohen, Doyle, Skoner, Rabin, \& Gwaltney, 1997a). Each item is a trait (bashful [-], shy [-], talkative, extraverted, quiet [-]), and respondents indicated how accurately the trait described how they "generally or typically are", as compared with other persons they know of the same sex and age, on a scale ranging from 0 (not at all accurate) to 4 (extremely accurate). The extraversion scale was administered twice, approximately 4 weeks apart, and the scores from the two assessments were averaged. The internal reliabilities for the two administrations were $.71-.78$ and the test-retest reliability $r=.81$, $p<.001$.

\section{Potential Mediating Variables}

We assessed smoking status and rate, alcohol consumption, exercise frequency, sleep duration and sleep efficiency as potential pathways mediating a link between subjective SES and colds. These behaviors were assessed in the 14 consecutive evening interviews described earlier. Each evening subjects were asked if they smoked any tobacco products, consumed any alcoholic drinks, or did exercise for long enough to work up a sweat and get their hearts thumping during the last 24 hours? Smokers (status) were defined as those who averaged at least one cigarette a day, and smoking rate as the square root of the average number of cigarettes smoked per day. Alcohol consumption was scored as the number of alcoholic drinks consumed on an average day, and exercise frequency as the number of days per week the subject was engaged in an activity long enough to "work up a sweat", "get the heart thumping", or "get out of breath" (Paffenbarger, Blair, Lee \& Hyde, 1993). Each evening they were also asked, "What time did you lie down to go to sleep last night?", "What time did you go to sleep last night?", "What time did you get out of bed this morning?" Finally, "How many minutes of sleep did you lose because you had difficulty falling asleep or woke up and couldn't get back to sleep?" Sleep duration was scored as the number of hours slept per night (from time the subject went to sleep until he/she got out of bed, minus minutes of sleep lost) and sleep efficiency as sleep duration divided by time in bed (time from lying down until getting out of bed) (cf. Monk et al., 1994). Both were averaged over the 14 days of data collection (or a minimum of 8 days for subjects missing interview data) to obtain average sleep duration and average sleep efficiency scores.

\section{Viral Cultures and Antibody Response}

Virus-specific neutralizing antibody titer was measured in serum collected before and approximately 28-days after virus exposure (Gwaltney, Colonno, Hamparian, \& Turner, 1989), and results were expressed as reciprocals of the final dilution of serum. Nasal lavage samples from each day were frozen at $-80^{\circ} \mathrm{C}$ and later cultured for rhinovirus or influenza virus using standard techniques (Gwaltney et al., 1989; Tobita, Sugiura, Enomoto, \& Furuyama, 1975).

\section{Signs and Symptoms}

On each day of quarantine, subjects rated the severity (during the previous 24 hours) of each of eight illness symptoms (nasal congestion, sneezing, runny nose, earache, sinus pain, sore throat, cough, and chest congestion) on a scale of 0 (none) to 4 (very severe) (Farr et al., 1990).

Daily mucus production was assessed by collecting used tissues in sealed plastic bags (Doyle, McBride, Swarts, Hayden, \& Gwaltney, 1988). The bags were weighed and the weight of the tissues and bags subtracted. Nasal mucociliary clearance function was assessed as the time required for dye administered into the anterior nose to reach the nasopharnyx (Doyle et al., 1988).

Baseline-adjusted daily scores for each measure were calculated by subtracting the appropriate baseline score from each of the post-exposure daily scores. Negative adjusted scores were reassigned a value of 0 . Total scores for symptoms, mucus weight, and nasal clearance were calculated by summing the respective adjusted daily scores over the quarantine days following viral exposure.

Volunteers were considered to have a clinical cold if they were both infected and met illness criteria. Infection was defined as recovery of the challenge virus on any of the post challenge days or $\mathrm{a} \geq$ four-fold rise in virus-specific serum neutralizing antibody titer (pre-exposure to 28-days post-exposure) (Cohen et al., 1997a; Cohen, Tyrrell, \& Smith, 1991). We used an objective criteria for 
illness that required a total adjusted mucus weight of at least 10 grams or a total adjusted mucociliary nasal clearance time of at least 35 minutes (Cohen et al., 1997a). For those with clinical colds by this criteria, the mean total adjusted respiratory symptom score was $31.00(S D= \pm 21.71)$ versus $8.56(S D= \pm 11.94)$ for those without colds $(t(191)=-9.25, p<.001)$.

\section{Statistical Analyses}

Scores for BMI, total symptoms, mucus weight, mucociliary clearance, and negative emotional style were logged (base-10) to better approximate a normal distribution. Logistic regression was used to predict colds (yes or no) and multiple linear regression was used to predict continuous markers of objective illness and total self-reported symptom scores. All SES measures were treated as continuous variables and we report regression coefficients, their standard errors, and probability levels. To illustrate the effect size of the association between subjective SES and colds, we also report the odds ratios and confidence intervals with the predictor split into tertiles.

\section{Results}

\section{Descriptive Data}

Table 1 presents descriptive data on all the variables involved in the analyses. Of 193 subjects, 157 (81.3\%) were infected, and 62 (32.1\%) developed clinical colds.

\section{Correlation of Objective and Subjective SES}

Subjective SES was correlated with education $(r=.17, p<.02)$ but not with income $(r=.06, p>.43)$. When both objective SES measures were entered into a linear regression equation predicting subjective SES, they accounted for $2.7 \%$ of the variance.

\section{Data Analysis}

We ran separate equations predicting clinical colds from each of the standard control variables. Those with greater levels of antibody were protected from developing illness $(b=-.78 \pm .31, p<$ .02 ) and those exposed to RV39 were more likely to develop a clinical illness than those exposed to the flu virus $(b=.89 \pm .45$, $p<.05)$. None of the remaining standard controls $(p>.14)$ predicted colds (Cohen et al., 2006).

All of the analyses include three covariates: virus, antibody level, and race. We included virus and antibody level because they are associated with risk for developing a cold and race because of the importance of distinguishing SES effects from those of race. Trimming the number of covariates avoids overfitting models. An unacceptable risk for overfitting in this sample for the logistic model would be more than 6 predictor variables [see Bagley, White, \& Golomb, 2001]). The major analyses are done using the trimmed covariate set. However, analyses including all of the standard controls yield the same results.

\section{SES}

Neither income nor education was associated with developing a clinical illness, with the objective signs of illness, nor with self-
Table 1

Descriptive Data for all Variables in the Equations

\begin{tabular}{|c|c|c|}
\hline & Mean & $S D$ \\
\hline \multicolumn{3}{|l|}{ Standard controls } \\
\hline Age (years) & 37.26 & 8.77 \\
\hline \multirow[t]{2}{*}{ BMI $(\mathrm{kg} / \mathrm{m} 2)$} & 28.98 & 7.08 \\
\hline & $N$ & $\%$ of total \\
\hline \multicolumn{3}{|l|}{ Season } \\
\hline Spring & 100 & 51.8 \\
\hline Summer & 14 & 7.3 \\
\hline Fall & 72 & 37.3 \\
\hline Winter & 7 & 3.6 \\
\hline \multicolumn{3}{|l|}{ Race } \\
\hline White / Caucasian & 108 & 56.0 \\
\hline Black / African American & 72 & 37.3 \\
\hline Other & 13 & 6.7 \\
\hline \multicolumn{3}{|l|}{ Sex } \\
\hline Female & 98 & 50.8 \\
\hline \multirow[t]{2}{*}{ Male } & 95 & 49.2 \\
\hline & Mean & $S D$ \\
\hline \multicolumn{3}{|l|}{ SES } \\
\hline Income $(\$)$ & 21753.93 & 20181.73 \\
\hline Education (years) & 13.76 & 2.21 \\
\hline Subjective SES & 4.77 & 1.95 \\
\hline \multicolumn{3}{|l|}{ Personal attributes } \\
\hline Mastery & 21.82 & 3.18 \\
\hline Optimism & 15.56 & 4.12 \\
\hline Self esteem & 13.81 & 1.80 \\
\hline Purpose (life engagement) & 25.09 & 3.58 \\
\hline Extraversion & 14.42 & 3.84 \\
\hline \multicolumn{3}{|l|}{ Emotional styles } \\
\hline $\begin{array}{l}\text { Trait positive affect (Positive emotional } \\
\text { style) }\end{array}$ & 14.94 & 4.04 \\
\hline $\begin{array}{l}\text { Trait negative affect (Negative } \\
\text { emotional style) }\end{array}$ & 3.22 & 2.86 \\
\hline \multicolumn{3}{|l|}{ Health practices - Daily interview } \\
\hline Alcoholic drinks per day & 1.26 & 2.52 \\
\hline Days of exercise per week & 2.43 & 1.90 \\
\hline Sleep efficiency & 0.94 & 0.06 \\
\hline Sleep duration (hours) & 7.40 & 1.25 \\
\hline \multirow[t]{2}{*}{ Smoking rate (cigarettes per day) } & 5.54 & 7.65 \\
\hline & $N$ & $\%$ of total \\
\hline Smoking status & & \\
\hline Averaged at least 1 per day & 92 & 47.7 \\
\hline
\end{tabular}

reported symptoms. In contrast, lower levels of subjective SES were associated with increasingly higher risk for developing a cold $(b=-.20$ [ \pm .08$], p<.02$ for continuous data; OR $=3.13$ [CI 1.38 to 7.10 ] for lowest tertile, 2.28 [CI 1.02 to 5.10] for middle, and 1 for highest; Figure 1). There was no association of race and cold incidence, nor was there a subjective SES-by-race interaction. In addition, neither the subjective SES-by-antibody nor the subjective SES-by-virus interactions entered the equation.

Analyses of the continuous data similarly found that lower levels of subjective SES were associated with more mucus production $(b=-.06[ \pm .02], p<.01)$ and with more total cold symptoms $(b=-.04[ \pm .02], p<.04)$. Subjective SES was not associated with mucociliary clearance time. 


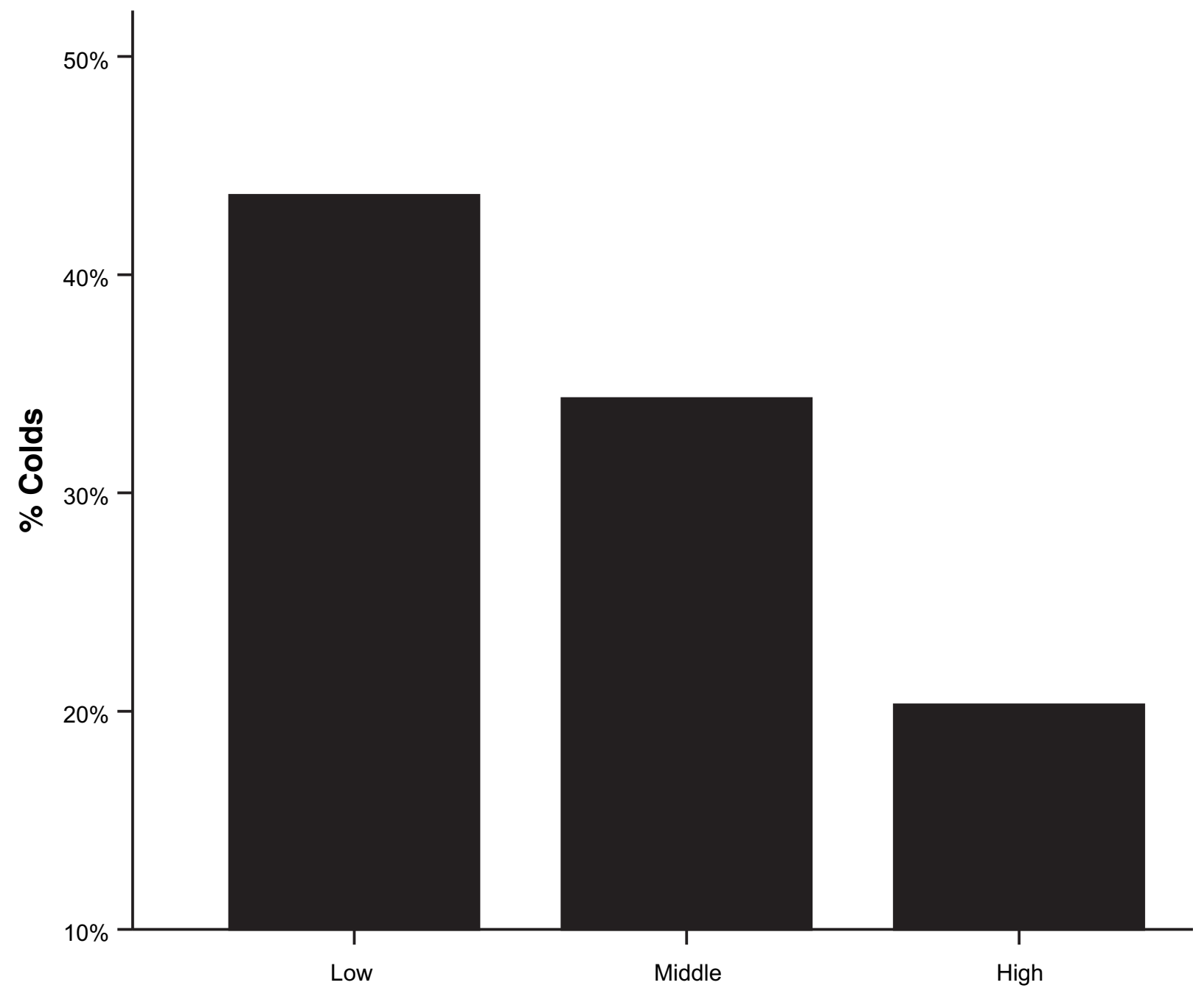

Subjective Socioeconomic Status (Ladder Score)

Figure 1. Percent of objectively verified colds by tertiles of subjective SES, adjusted for race, virus, and pre-challenge antibody level. Adjusted scores for each individual are created by adding the residual score to the predicted value.

Because our definition of clinical colds combines infection with illness, the observed association between lower subjective SES and clinical colds could have resulted from an increased risk for infection and/or an increased expression of illness among infected persons. Subjective SES was not associated with infection rates $(p>.97)$, but lower ladder scores were associated with increased rates of clinical colds among infected subjects $(b=-.22$ [ \pm .09$]$, $p<.02 ; \mathrm{OR}=3.84$ [CI 1.60 to 9.24 ] for lowest tertile, 2.38 [CI 1.02 to 5.53] for middle, and 1 for highest). Hence, the relation between subjective SES and colds is attributable to infected people with lower subjective SES expressing more objective signs of illness. Separate analyses of the association of subjective SES with the continuous measures of cold signs and self-reported symptoms in infected subjects were consistent with this association; for mucus weights, $b=-.07[+.03], p<.01$; and for symptoms, $b=$ $-.06[+.02], p<.02$. There was no significant association with mucociliary clearance function $(p>.98)$.
In an additional analysis, we added the objective measures of SES, income and education, as controls to these same equations. The results were unchanged. A lower level of subjective SES was associated with higher risk for developing a cold $(b=-.23$ [ \pm .09$], p<.02$ for continuous analysis; OR $=3.65$ [CI 1.55 to 8.59] for lowest tertile, 2.44 [CI 1.07 to 5.58] for middle, and 1 for highest; greater mucus production $(b=-.06[ \pm .02], p<.01)$; and more total symptoms $(b=-.04$ [ \pm .02$], p<.05)$. It was not associated with clearance function.

\section{Possible Confounders and Mediators}

We then fit a series of logistic regression models predicting colds. Each included virus, antibody level, race, subjective SES and one of the hypothesized mediators or confounders. The third factor explanations (confounders) of the association between subjective SES and colds we tested for included negative emotional 
style, positive emotional style, self-esteem, mastery, purpose, optimism, and extraversion. Finally we fit a single model including all of these control variables at once. The association between subjective SES and colds was maintained in all of these equations $(P$ s range from $<.02$ to $<.03)$.

We also thought that health practices might operate as mediating pathways linking subjective SES to colds. We fit another series of regression equations as above, adding in individual models the various health practices: smoking status, smoking rate, alcohol consumption, exercise, sleep duration, and sleep efficiency. The association between subjective SES and colds was maintained in the equations adding smoking status, smoking rate, alcohol consumption and exercise (Ps range from $<.01$ to $<.04$ ). However, when sleep efficiency was added to the equation the effect size (beta) was reduced $20 \%$, and when sleep duration was added it was reduced $16 \%$. With both added, the association of subjective SES and colds was reduced to $b=-.14[+.09], p<.18$, a reduction in effect size of $28 \%$. These data are consistent with these markers of sleep quality, partly mediating the association between subjective SES and susceptibility.

\section{Discussion}

Like earlier work on subjective SES, we found that the ladder score predicted health independent of objective markers of SES. However, unlike earlier studies, this study was prospective, eliminating the possibility that illness might influence a person's perceptions of their social status. It also involved a hard (objective) outcome; demonstrating that subjective SES has implications for risk of physical disease and eliminating the possibility that existed in many of the earlier studies that a response bias influenced both reports of subjective SES and health outcomes.

Unlike much of the literature on SES and other disease outcomes, we did not find an association between objective markers of SES and cold susceptibility. This is likely because the distribution of objective markers (especially income) in this sample is quite unusual. Because the sample is made up of volunteers who are willing to be quarantined for almost a week, and who get paid for their participation, the sample is somewhat skewed to the lower end. More importantly, it is unlikely that the better educated people in the sample provide an unbiased representation of the higher levels of SES. In fact, it is possible that at least some of the higher educated subjects in our sample are motivated to volunteer because their incomes and occupations are not commensurate with their educations.

Why would levels of subjective status differ substantially from status inferred from the objective markers? A number of explanations are based on the possibility that the subjective status measure just does a better job of measuring social standing. First, subjective social status may reflect the success or failure to meet one's educational potential, while the objective measures do not. Second, subjective status allows the respondent to weigh income, education, and occupation in proportion to the importance of each marker in the respondent's own social context. For example, education may be a more important determinant of status for a college professor and income for an entrepreneur. Third, the objective measures of SES are crude and the ladder may be capturing finer gradations of the objective indicators than the objective markers. For example, years of education does not distinguish between the quality and status of the school attended (e.g., community college versus Ivy League school), but the respondents know that education scores have different meanings depending on school. Finally, subjective ratings probably capture a broader range of SES markers, including wealth, living locations and conditions, and parental SES, that are not measured by our more limited range of objective markers.

On the other hand, it is possible that subjective SES is not tightly associated with objective SES because the ladder is not merely assessing perceived status. At least to some degree, subjective SES may represent characteristics of individuals that bias their perceptions of their social status. Variables that have a history of being associated with health outcomes and might influence a biased perception include negative and positive emotional styles, personal control, self-esteem, optimism, and purpose in life. However, controlling for these variables did not influence the association between subjective SES and colds.

What is it that makes those lower in subjective status more susceptible and those higher more resilient? Our data suggest that subjective status may partly operate through sleep duration and efficiency. It could be that those low in subjective status worry more (although we find no influence of general negative affect) or feel a need to be more vigilant, which then influences their ability to sleep and, consequently, their susceptibility to disease. Alternatively, subjective social status may have direct influences on biological processes that interfere with sleep.

What about mediators other than sleep? The data do not support the hypothesis that a number of commonly suggested psychological variables operate as pathways, including selfesteem, mastery, purpose, optimism, affect, and extraversion. (Controlling for these variables does not reduce the association between the ladder and colds.) However, it is possible that some other psychological characteristics not measured here, such as social support, prestige, power, or relative deprivation, may also play a role. Moreover, it could be that subjective social rank is a sufficient psychological condition (independent of other psychological characteristics) to drive whatever biological pathways are responsible here. Finally, recent evidence that subjective, but not objective, social status is associated with reduced gray matter volume in a brain region involved in regulating behavioral and physiological reactivity to psychosocial stress-perigenual area of the anterior cingulate cortex (Gianaros et al., 2007) suggests that better assessment of the ability to cope with stress and regulate emotional responses might reveal an additional pathway.

In sum, we found that subjective, but not objective, social status was associated with disease susceptibility. It is likely that at least part of this association was mediated by poorer sleep duration and efficiency with lesser subjective status.

\section{References}

Adler, N. E., Boyce, T., Chesney, M. A., Cohen, S., Folkman, S., Kahn, R. L., et al. (1994). Socioeconomic status and health: The challenge of the gradient. American Psychologist, 49, 15-24.

Adler, N. E., Epel, E. S., Castellazzo, G., \& Ickovics, J. R. (2000). Relationship of subjective and objective social status with psychological and physiological functioning: Preliminary data in healthy white women. Health Psychology, 19, 586-592. 
Anderson, N. B., \& Armstead, C. A. (1995). Toward understanding the association of socioeconomic status and health: A new challenge for the biopsychosocial approach. Psychosomatic Medicine, 57, 213-225.

Bagley, S. C., White, H., \& Golomb, B. A. (2001). Logistic regression in the medical literature: Standards for use and reporting, with particular attention to one medical domain. Journal of Clinical Epidemiology, 54, 979-985.

Cohen, S., Alper, C. M., Doyle, W. J., Treanor, J. J., \& Turner, R. B. (2006). Positive emotional style predicts resistance to illness after experimental exposure to rhinovirus or influenza A virus. Psychosomatic Medicine, 68, 809-815.

Cohen, S., Doyle, W. J., Skoner, D. P., Rabin, B. S., \& Gwaltney, J. M., Jr. (1997a). Social ties and susceptibility to the common cold. Journal of the American Medical Association, 277, 1940-1944.

Cohen, S., Line, S., Manuck, S. B., Rabin, B. S., Heise, E., \& Kaplan, J. R. (1997b). Chronic social stress, social status and susceptibility to upper respiratory infections in nonhuman primates. Psychosomatic Medicine, 59, 213-221.

Cohen, S., Tyrrell, D. A. J., \& Smith, A. P. (1991). Psychological stress and susceptibility to the common cold. New England Journal of Medicine, 325, 606-612.

Doyle, W. J., McBride, T. P., Swarts, J. D., Hayden, F. G., \& Gwaltney, J. M., Jr. (1988). The response of the nasal airway, middle ear and Eustachian tube to provocative rhinovirus challenge. American Journal of Rhinology, 2, 149-154.

Farr, B. M., Gwaltney, J. M., Jr., Hendley, J. O., Hayden, F. G., Naclerio, R. M., McBride, T., et al. (1990). A randomized controlled trial of glucocorticoid prophylaxis against experimental rhinovirus infection. Journal of Infectious Diseases, 162, 1173-1177.

Gianaros, P. J., Horenstein, J. A., Cohen, S., Matthews, K. A., Brown, S. M., Flory, D., Critchley, H. D., Manuck, S. B., \& Hariri, A. R. (2007). Perigenual anterior cingulate morphology covaries with perceived social standing. Social Cognitive and Affective Neuroscience, 2, 161-173.

Goldberg, L. R. (1992). The development of markers for the Big-Five factor structure. Psychological Assessment, 4, 26-42.

Gwaltney, J. M., Jr., Colonno, R. J., Hamparian, V. V., \& Turner, R. B. (1989). Rhinovirus. In N. J. Schmidt \& R. W. Emmons (Eds.), Diagnostic procedures for viral, rickettsial and chlamydial infections (6th ed., pp. 579-614). Washington, DC: American Public Health Association.

Hu, P., Adler, N. E., Goldman, N., Weinstein, M., \& Seeman, T. E. (2005). Relationship between subjective social status and measures of health in older Taiwanese persons. Journal of the American Geriatrics Society, 53, 483-488.

Kaplan, J. R., Manuck, S. B., Clarkson, T. B., Lusso, F. M., \& Taub, D. B. (1982). Social status, environment and atherosclerosis in cynomolgus monkeys. Arteriosclerosis, 2, 359-368.

Marmot, M., Feeney, A., Shipley, M., North, F., \& Syme, S. L. (1995). Sickness absence as a measure of health status and functioning: From the UK Whitehall II study. Journal of Epidemiology \& Community Health, 49, $124-130$.

Monk, T. H., Reynolds, C. F. III, Kupfer, D. J., Buysse, D. J., Coble, P. A., Hayes, A. J., Machen, M. A., Petrie. S. R., \& Ritenour, A. M. (1994). The Pittsburgh Sleep Diary. Journal of Sleep Research, 3, 111-120.

Ostrove, J. M., Adler, N. E., Kuppermann, M., \& Washington, A. E. (2000). Objective and subjective assessments of socioeconomic status and their relationship to self-rated health in an ethnically diverse sample of pregnant women. Health Psychology, 19, 613-618.

Paffenbarger, R. S. Jr., Blair, S. N., Lee, I., \& Hyde, R. T. (1993). Measurement of physical activity to assess health effects in free-living populations. Medicine and Science in Sports and Exercise, 25, 60-70.

Pearlin, L. I., \& Schooler, C. (1978). The structure of coping. Journal of Health and Social Behavior, 19, 2-21.

Rosenberg, M. (1965). Society and the adolescent self-image. Princeton, NJ: Princeton University Press.

Scheier, M. F., Carver, C. S., \& Bridges, M. W. (1994). Distinguishing optimism from neuroticism (and trait anxiety, self-mastery, and selfesteem): A reevaluation of the Life Orientation Test. Journal of Personality and Social Psychology, 67, 1063-1078.

Scheier, M. F., Wrosch, C., Baum, A., Cohen, S., Martire, L. M., Matthews, K. A., et al. (2006). The Life Engagement Test: Assessing purpose in life. Journal of Behavioral Medicine, 29, 291-298.

Singh-Manoux, A., Adler, N. E., \& Marmot, M. G. (2003). Subjective social status: Its determinants and its association with measures of ill-health in the Whitehall II study. Social Science \& Medicine, 56, 1321-1333.

Tobita, K., Sugiura, A., Enomoto, C., \& Furuyama, M. (1975). Plaque assay and primary isolation of influenza A viruses in an established line of canine kidney cells (MDCK) in the presence of trypsin. Medical microbiology and immunology (Berl), 162, 9-14.

Wright, C. E., \& Steptoe, A. (2005). Subjective socioeconomic position, gender and cortisol responses to waking in an elderly population. Psychoneuroendocrinology, 30, 582-590. 